\title{
Validação de um questionário de atitude em relação à soja e seus derivados com clientes da alimentação coletiva
}

\author{
Validation of a questionnaire to assess food \\ service customer's attitude towards \\ soybeans and its products
}

Silvia Magalhães COUTO'

Gabriela Morgado de Oliveira COELHO²

Alessandra Silva de SOUZA²

Marina de Figueiredo FERREIRA²

Victor Augustus MARIN ${ }^{3}$

Haydée Serrão LANZILLOTTI ${ }^{4}$

R E S U M O

\section{Objetivo}

Validar um questionário para verificar a atitude de clientes da Alimentação Coletiva em relação à soja e seus derivados.

\section{Métodos}

A elaboração do questionário valeu-se de escala de Likert de cinco pontos. As validações foram realizadas com clientes de duas unidades de alimentação e nutrição, da cidade do Rio de Janeiro, sendo a primeira uma empresa gráfica e a segunda uma empresa de confecções. A validação interna valeu-se do coeficiente de Cronbach para medir confiabilidade. Aplicou-se o teste de normalidade às matrizes de escores para cada item. A constatação do comportamento dos escores não Gausiano indicou utilização da correlação de Spearman $(r)$ para discriminar itens. Como critério de exclusão foi definido $r$ negativo ou próximo de zero. A validação externa foi realizada entre o confronto das matrizes representadas pelas médias dos escores de cada item. Utilizou-se a razão dos desvios-padrão para avaliar a concordância relativa, considerando-se a associação entre as matrizes resultantes da aplicação do questionário nas duas unidades de alimentação e nutrição.

1 Universidade Federal do Rio de Janeiro, Centro de Ciências da Saúde. Instituto de Nutrição Josué de Castro. Av. Carlos Chagas Filho, 373, Bloco J, $2^{\circ}$ andar, 21941-590, Ilha do Fundão, RJ, Brasil. Correspondência para/Correspondence to: S.M. COUTO. E-mail: <silviacouto@globo.com>.

2 Universidade Gama Filho, Curso de Nutrição. Rio de Janeiro, RJ, Brasil.

3 Fundação Oswaldo Cruz, Instituto Nacional de Controle de Qualidade em Saúde. Rio de Janeiro, RJ, Brasil.

${ }^{4}$ Universidade do Estado do Rio de Janeiro, Instituto de Nutrição. Rio de Janeiro, RJ, Brasil. 
632 | S.M. couto et al.

\section{Resultados}

Participaram da validação 45 e 90 clientes das duas unidades de alimentação e nutrição, respectivamente. O procedimento aplicado na unidade de alimentação e nutrição da empresa gráfica indicou a retirada de 3 itens dentre os 20 itens originais. O questionário resultante foi replicado na unidade de alimentação e nutrição da empresa de confecção, sem indicação de exclusão de itens. O coeficiente de Cronbach foi 0,92 e 0,91, respectivamente, indicando confiabilidade do instrumento para medir atitude. O confronto entre os escores para cada item entre as unidades gerou a razão de desvio 0,99, que recomenda retirada do item seis, relativo à rotulagem, ou seja, se as informações são suficientemente claras em relação à soja.

\section{Conclusão}

O questionário foi validado, tornando-se um instrumento capaz de informar sobre a atitude de clientes da alimentação coletiva em relação à soja e seus derivados.

Termos de indexação: Alimentação coletiva. Feijão de soja. Questionário.

\section{A B S T R A C T}

\section{Objective}

The objective was to validate a questionnaire that verifies food service customers' attitude towards soybeans and its products.

\section{Methods}

A five-point Likert scale was used to develop the questionnaire. Validation was accomplished with customers of two food and nutrition units in the city of Rio de Janeiro, the first being a printing company and the second a clothing company. Cronbach's alpha was used to measure internal reliability. The normality test was applied to the score matrices of each item. The identification of scores with non-Gaussian behavior indicated the need to use Spearman's correlation (r) to discriminate items. The exclusion criterion was correlations with negative or close to zero $r$. The external validation was established by confronting the matrices represented by the mean scores of each item. The standard deviation ratio was used to assess the relative agreement considering the association between the matrices which resulted from the administration of the questionnaires in both Food and Nutrition Units.

\section{Results}

A total of 45 and 90 clients from the two food and nutrition units respectively participated in the validation process. The procedure administered the printing company unit indicated the removal of three items from the twenty original items. The resulting questionnaire was administered in the clothing company unit, with no indication of item removal. The Cronbach's coefficient was 0.92 and 0.91 , respectively showing that the instrument measures attitude reliably. The confrontation between the scores of each item and between the two food and nutrition units generated a deviation ratio of 0.99 , which recommends the removal of item number six, regarding labeling, that is, if the information regarding soybeans is sufficiently clear.

\section{Conclusion}

The questionnaire was validated, therefore it is an instrument capable of determining food service customers' attitude towards soybeans and its products.

Indexing terms: Food services. Soybeans. Questionnaire.

\section{N T R O D U Ç Ã O}

O comportamento alimentar e o estilo alimentar são dimensões a ter em conta em alimentação coletiva. São poucos os trabalhos que se dedicam à construção de instrumentos adequados para a investigação destes fatores. Neste sentido, torna-se urgente criá-los ou adaptá-los, no entanto exige-se que tenham valor reconhecido entre a comunidade científica. Nos dias de hoje, existe um interesse crescente em consumir alimentos mais saudáveis, o que tem conduzido a indústria alimentícia ao desenvolvimento de produtos utilizando alimentos funcionais, dentre eles a soja e seus derivados, seja sob a forma de preparação alimentar ou de ingredientes de produtos alimen- 
tares. Existem estratégias de marketing para inserir esses produtos no mercado. Frente a isso, faz-se necessário conhecer a atitude dos clientes da Alimentação Coletiva sobre o uso desses alimentos neste setor produtivo.

"Atitude é uma predisposição aprendida pelo indivíduo, formada a partir de suas experiências e informações obtidas, as quais o influenciam a agir favorável ou desfavoravelmente em relação a um determinado objeto. Este objeto pode ser um produto ou categoria de produtos, marcas, serviços, bens, pessoas, conceitos/idéias, propaganda, preço, canais de comércio, mídia, entre outros"1 (p.432).

A partir de meados do século passado o padrão de consumo, principalmente nos países desenvolvidos tem se alterado em função das novas preocupações com saúde, qualidade de vida e meio ambiente, por parte dos consumidores.

A biotecnologia representa um novo paradigma na produção de alimentos e, conseqüentemente, na alimentação coletiva, uma vez que guarda um grande potencial para a produção de alimentos com melhores propriedades nutricionais e sensoriais. Numerosas podem ser as alterações possíveis em benefício da segurança alimentar proporcionada pela biotecnologia, e passíveis de serem incluídas na rotina da alimentação coletiva. Alguns exemplos a serem citados são: alimentos: cenoura (aumento de carotenóides), batata (maior teor de amido e aumento da proporção de tirosina), brócolis (maior tempo de vida útil), tomate e frutas (amadurecimento retardado) ${ }^{2}$.

A polêmica a respeito destes produtos ocorre em muitos níveis diferentes: científico, técnico, político, econômico, social e filosófico, todos entrelaçados e difíceis de distinguir ${ }^{3}$.

Duas razões podem ser avocadas em favor da liberação dos produtos transgênicos, em especial a soja, na agricultura brasileira. A primeira relacionada ao fato de ser uma tecnologia de ponta, incorporada à pesquisa nacional, como decorrência dos intercâmbios científicos com nações desenvolvidas e a segunda razão, por ser uma tecnologia com presumíveis vantagens econômicas a seus clientes. Esta situação induziria a que, por força da pressão das empresas (e dos agricultores), mais cedo ou mais tarde, houvesse sua adoção. Conseqüentemente, a liberação dos produtos transgênicos seria parte do avanço natural das descobertas científicas e das inovações tecnológicas. De outra parte, entende-se ser inócua uma proibição, dado o fato de que o Brasil já estaria cercado e invadido por transgênicos, presentes, em maior ou menor grau, em produtos americanos e argentinos industrializados, importados, além de lavouras plantadas no Sul do País, com sementes transgênicas contrabandeadas da Argentina. Leis estaduais e municipais restritivas à comercialização de produtos transgênicos embasam ações de apreensão de produtos importados, sejam eles nacionais ou que contenham matéria-prima importada com Organismo Geneticamente Modificado (OGM). Organizações da sociedade civil (como Instituto Brasileiro de Defesa do Consumidor - IDEC) promovem análises e denunciam a comercialização desses produtos, a qual pode ser considerada ilegal, segundo uma interpretação mais rigorosa ${ }^{4}$.

No Brasil, a utilização e o comércio de transgênicos são regulamentados pela Comissão Técnica Nacional de Biossegurança (CTNBio), órgão criado pela Lei 8.974 , de 5 de janeiro de 1995, e reestruturado pela Lei 11.105, de 24 de março de 2005. Assim, é assegurado o direito à informação quanto aos alimentos e aos ingredientes alimentares destinados ao consumo humano ou animal que contenham ou sejam produzidos a partir de organismos geneticamente modificados $^{5}$.

Parte-se da hipótese de que não há acesso da população sobre informações relativas à presença da soja, talvez transgênica, explicitada ou não nos rótulos de alimentos processados. Não sendo refutada tal hipótese, indivíduos podem estar consumindo soja com freqüência em vários alimentos e preparações alimentares sem ter conhecimento do que consomem. Diante dessa possibilidade, o estudo tem como objetivo validar um questionário para verificar a atitude do cliente da Alimentação Coletiva em relação à soja e seus derivados. 


\section{MÉ TO D OS}

Unidade de Alimentação e Nutrição (UAN): uma Empresa Gráfica (EG) e uma Empresa de Confecções (EC), ambas com, aproximadamente, 200 clientes, adotando atendimento self service em serviço terceirizado, localizadas na cidade do Rio de Janeiro, em 2006.

As validações interna e externa do questionário de atitude em relação à soja e seus derivados foram realizadas com clientes das duas UAN visitadas. A validação interna valeu-se da metodologia proposta por Behrens \& Da Silva ${ }^{1}$ para compor um questionário de pesquisa de atitude com relação à soja, utilizando escala de Likert de sete pontos. Neste estudo utilizou-se uma escala de Likert de cinco pontos (concordo muito a discordo muito), conforme o sugerido por Freeman ${ }^{6}$.

A opção por Freeman ocorreu em razão de os clientes da Alimentação Coletiva não estarem habituados a responder questionários, diferentemente dos estudantes universitários que participaram do estudo Behrens \& Silva ${ }^{1}$. Os clientes da Alimentação Coletiva teriam maior dificuldade de exprimir atitude com o gradiente de concordância mais complexo, como ocorre na escala de Likert de sete pontos, segundo a qual existe uma diferença muito tênues de concordância e discordância.

Foi realizado o teste de Kolmogorov-Smirnov para verificar o comportamento gausiano das matrizes dos escores de cada item. O coeficiente de correlação de Spearman ( $r$ ) discriminou os itens, ao verificar a associação entre as notas que os respondentes deram para cada item com a soma das notas obtidas por todos os itens da escala, conforme o sugerido por Müeller ${ }^{7}$. O critério de exclusão foi existir item com $r$ negativo ou próximo de zero. Uma correlação negativa representa uma direção oposta entre as notas atribuídas para cada item e o total dos itens, tornando frágil a validação interna. Em relação às correlações positivas, foi necessário aplicar a prova de significância para a correlação de Spearman, que utiliza a hipótese de nulidade, de que os itens não são associados na população e que o valor observado desta correlação difere de zero, por acaso. Segundo Siegel ${ }^{8}$, em grandes amostras esta prova utiliza a distribuição $t$ de Student, com grau de liberdade $n-2$. No presente trabalho, foi aplicado este procedimento e considerou-se nível de confiabilidade de $95 \%$ como o norteador das decisões quanto à permanência ou a exclusão dos itens. A medida de confiabilidade utilizada foi o coeficiente de Cronbach.

Para a validação externa do questionário aplicado nas UAN utilizou-se o método de Altman \& Bland ${ }^{9}$, que se fundamenta na premissa de que não existe relação óbvia entre as diferenças dos valores obtidos de cada método para cada sujeito e a sua respectiva média. Sob esta circunstância, a falta de concordância pode ser identificada calculando-se os vieses, estimados pela média das diferenças e o respectivo desvio-padrão. Um diagrama de dispersão permite traçar os limites de concordância estimados. Com a razão dos desvios obtida a partir da divisão entre o Desvio-Padrão das Diferenças (d.p.d.) sobre o Desvio-Padrão das Médias (d.p.m.) de ambas as UAN, avaliou-se a concordância relativa dos resultados obtidos em cada UAN. Quanto menor é a razão maior é a concordância relativa entre as aplicações do questionário ${ }^{10}$. As médias, obtidas nas duas UAN estudadas, foram utilizadas para verificar a tendência de concordância ou discordância com os itens do questionário, que media a atitude dos clientes em relação à soja e seus derivados.

A tendência de concordância, discordância ou estado de dúvida foi realizada utilizando seis categorias de análise, a saber: transgenia, valor funcional, comercialização, qualidade, rotulagem e valor nutricional.

O banco de dados foi criado em planilha Excel, incluindo análises da estatística descritiva. Para a aplicação do teste de Kolmogorov-Smirnov, a correlação de Spearman e o diagrama de Altman \& Bland ${ }^{9}$ foi utilizado o aplicativo Graph Pad Prism versão 4.

A pesquisa foi aprovada pelo Comitê de Ética em Pesquisa Envolvendo Seres Humanos da Universidade Gama Filho, protocolo CEPq/UGF 
009/jul/2006, observando-se o cumprimento dos preceitos éticos contidos na declaração de Helsinki, da World Medical Association. De cada entrevistado foi obtido o Consentimento Livre e Esclarecido. Todos os participantes foram previamente esclarecidos sobre o objetivo do trabalho e as técnicas as quais seriam submetidos, e só fizeram parte da amostra os que concederam a permissão para ser entrevistado.

\section{RESULTADOS}

Participaram da validação interna 45 clientes da UAN de uma empresa gráfica e da externa 90 clientes de UAN de uma empresa de confecções.

O teste de Kolmogorov- Smirnov aplicado às matrizes dos escores obtidos para cada item ao questionário revelou comportamento não gaussiano em ambas as UAN. Esta situação indicou o uso do coeficiente de Spearman para discriminação dos itens.

O procedimento de validação interna revelou que dos 20 itens originais (vide questionário em anexo), 3 itens apresentaram índice de discriminação negativo ou próximo a zero, ou seja, o item 5 (a difícil digestão da soja reduz seu consumo), o item 12 (deveria ser obrigatório pela legislação brasileira, declarar no rótulo o uso de soja trangênica em produtos industrializados) e o item 16 (os consumidores deveriam procurar as informações sobre os benefícios da soja na rotulagem). Esses itens foram eliminados do questionário, pois diminuíam o poder de discriminação e a confiabilidade da escala, sendo esta última avaliada pelo coeficiente de Cronbach, que atingiu valor de 0,92 (Tabela 1).

Tabela 1. Proporção das respostas (\%) e escore médio dos itens da escala de atitude em relação à soja e seus derivados, em uma unidade de alimentação e nutrição de uma empresa gráfica localizada na cidade do Rio de Janeiro (RJ), 2006.

\begin{tabular}{|c|c|c|c|c|c|c|c|c|c|}
\hline Itens & $1(\%)$ & $2(\%)$ & $3(\%)$ & $4(\%)$ & $5(\%)$ & Média & IC & ID & $p$ valor \\
\hline \multicolumn{10}{|c|}{ Itens retirados } \\
\hline 5. & 16 & 4 & 29 & 31 & 20 & 3,36 & 0,38 & 0,05 & $0,7466^{*}$ \\
\hline 12. & 58 & 31 & 11 & 0 & 0 & 1,53 & 0,20 & $-0,14$ & $0,3585^{*}$ \\
\hline 16. & 53 & 40 & 4 & 2 & 0 & 1,56 & 0,20 & $-0,01$ & $0,9192^{*}$ \\
\hline \multicolumn{10}{|c|}{ Itens positivos } \\
\hline 1. & 4 & 7 & 2 & 33 & 53 & 4,24 & 0,32 & 0,58 & 0,0001 \\
\hline 2. & 2 & 7 & 2 & 53 & 36 & 4,13 & 0,27 & 0,42 & 0,0044 \\
\hline 3. & 2 & 9 & 7 & 40 & 42 & 4,11 & 0,30 & 0,31 & 0,0416 \\
\hline 4. & 7 & 7 & 18 & 36 & 33 & 3,82 & 0,34 & 0,18 & $0,2366^{*}$ \\
\hline 6. & 18 & 31 & 33 & 9 & 9 & 2,60 & 0,34 & 0,22 & $0,1501^{*}$ \\
\hline 7. & 2 & 0 & 11 & 38 & 49 & 4,31 & 0,25 & 0,35 & 0,0179 \\
\hline 9. & 0 & 4 & 22 & 36 & 38 & 4,07 & 0,26 & 0,11 & $0,4566^{*}$ \\
\hline 10. & 0 & 4 & 60 & 16 & 20 & 3,51 & 0,25 & 0,14 & $0,3694^{*}$ \\
\hline 13. & 0 & 11 & 2 & 36 & 51 & 4,27 & 0,28 & 0,27 & 0,0781 \\
\hline 17. & 4 & 20 & 11 & 38 & 27 & 3,62 & 0,35 & 0,32 & 0,0308 \\
\hline 19. & 24 & 29 & 29 & 9 & 9 & 2,49 & 0,36 & 0,14 & $0,3606^{*}$ \\
\hline 20. & 0 & 0 & 13 & 40 & 47 & 4,33 & 0,21 & 0,53 & 0,0002 \\
\hline \multicolumn{10}{|c|}{ Itens negativos } \\
\hline 8. & 20 & 13 & 13 & 36 & 18 & 3,18 & 0,41 & 0,27 & 0,0683 \\
\hline 11. & 11 & 33 & 29 & 16 & 11 & 3,82 & 0,34 & 0,37 & 0,0117 \\
\hline 14. & 9 & 40 & 29 & 16 & 7 & 2,71 & 0,31 & 0,16 & $0,3000^{*}$ \\
\hline 15. & 13 & 40 & 18 & 24 & 4 & 2,67 & 0,33 & 0,25 & 0,0966 \\
\hline 18. & 18 & 38 & 33 & 4 & 7 & 2,44 & 0,31 & 0,51 & 0,0004 \\
\hline
\end{tabular}

${ }^{*}$ ) Significante (n.45).

IC: intervalo de confiança $(p<0,05)$; ID: índice de discriminação.

Escala de Likert: 1 discordo muito a 5 concordo muito para os itens positivos e o inverso para os itens negativos. 
A probabilidade de decidir que haja associação entre as notas atribuídas ao item 5 em relação à soma das notas atribuídas aos demais itens $\left(r_{s}=0,05\right)$ correspondeu a $76 \%$, portanto é viável acreditar que a correlação esteja bem próxima de zero. O item 9 seria o próximo com probabilidade de risco de $45 \%$, defasagem de $31 \%$, bem distanciada do item anterior. Justifica-se eliminar apenas o item 5. Os itens 5, 12 e 16 não foram inseridos na replicação do questionário.

Frente à indicação da retirada de três itens, fez-se necessário aplicar o questionário na UAN da EC para replicar o procedimento. Os dados encontram-se consolidados na Tabela 2 (Questionário em anexo).

Replicado o mesmo procedimento na UAN de EC, ocorreu que o item 9 apresentou maior risco $(61 \%), 15 \%$ pontos percentuais aquém do item 5 do questionário aplicado na UAN EG, tendo-se julgado pertinente a sua permanência na categoria de valor nutricional. Acresce-se ainda que a aferição do aporte protéico da soja é relevante, pois em razão deste aporte, esta leguminosa se torna matéria-prima para obtenção de vários derivados protéicos, como a farinha (integral e desengordurada), os isolados e concentrados protéicos, a proteína vegetal texturizada e os extratos hidrossolúveis, que serão utilizados pela indústria com diversas finalidades tecnológicas na elaboração de alimentos processados.

O coeficiente de Cronbach alcançou valor de 0,91 . Os achados permitem inferir que os questionários, o primeiro com 20 itens e o segundo com 17 itens, podem ser considerados confiáveis.

A validação externa foi realizada utilizando-se os dados oriundos da aplicação em EG, retirando-se os 3 itens discriminados e os resultantes da aplicação em EC, com 17 itens.

O diagrama de Altman \& Bland ${ }^{9}$, gerado com os resultados da aplicação dos questionários

Tabela 2. Proporção das respostas (\%) e escore médio dos itens da escala de atitude em relação à soja e seus derivados, em Unidade de Alimentação e Nutrição de uma empresa de confecções localizada na cidade do Rio de Janeiro (RJ), 2006.

\begin{tabular}{|c|c|c|c|c|c|c|c|c|c|}
\hline Itens & $1(\%)$ & $2(\%)$ & $3(\%)$ & $4(\%)$ & $5(\%)$ & Média & IC & ID & $p$ valor \\
\hline \multicolumn{10}{|c|}{ Itens positivos } \\
\hline 1. & 9 & 7 & 2 & 24 & 58 & 4,16 & 0,27 & 0,40 & 0,0001 \\
\hline 2. & 8 & 6 & 0 & 23 & 63 & 4,29 & 0,25 & 0,29 & 0,0053 \\
\hline 3. & 6 & 3 & 9 & 17 & 66 & 4,33 & 0,23 & 0,39 & 0,0001 \\
\hline 4. & 7 & 10 & 10 & 12 & 61 & 4,11 & 0,27 & 0,42 & 0,0001 \\
\hline 6. & 13 & 28 & 34 & 6 & 19 & 2,89 & 0,26 & 0,35 & 0,0007 \\
\hline 7. & 32 & 18 & 18 & 17 & 16 & 2,72 & 0,32 & 0,23 & 0,0291 \\
\hline 9. & 1 & 4 & 12 & 30 & 52 & 4,28 & 0,19 & 0,05 & $0,6121^{*}$ \\
\hline 10. & 0 & 8 & 73 & 8 & 11 & 3,22 & 0,15 & 0,14 & $0,1659^{*}$ \\
\hline 13. & 2 & 1 & 0 & 18 & 79 & 4,70 & 0,15 & 0,23 & 0,0311 \\
\hline 14. & 11 & 12 & 33 & 19 & 24 & 3,33 & 0,26 & 0,08 & 0,4630 \\
\hline 19. & 6 & 10 & 36 & 27 & 22 & 3,50 & 0,23 & 0,22 & 0,0404 \\
\hline 20. & 3 & 4 & 10 & 13 & 69 & 4,40 & 0,22 & 0,29 & 0,0058 \\
\hline \multicolumn{10}{|c|}{ Itens negativos } \\
\hline 8. & 30 & 22 & 42 & 0 & 6 & 2,29 & 0,22 & 0,37 & 0,0003 \\
\hline 11. & 31 & 18 & 19 & 17 & 16 & 2,68 & 0,30 & 0,14 & $0,1659^{*}$ \\
\hline 17. & 6 & 11 & 34 & 21 & 28 & 3,54 & 0,24 & 0,06 & $0,5966^{*}$ \\
\hline 15. & 24 & 10 & 28 & 17 & 21 & 3,00 & 0,30 & 0,07 & $0,5320^{*}$ \\
\hline 18. & 10 & 12 & 37 & 8 & 33 & 3,42 & 0,28 & 0,40 & 0,0001 \\
\hline
\end{tabular}

(*) Significante (n.90).

IC: intervalo de confiança $(p<0,05)$; ID: índice de discriminação.

Escala de Likert: 1 discordo muito a 5 concordo muito para os itens positivos e o inverso para os itens negativos. 
nas UAN EG e EC (Figura 1), ambos com 17 itens, mostrou que $94,12 \%$ das diferenças entre os escores distribuíam-se entre os limites de concordância (Tabela 3). A razão dos desvios $(0,64 / 0,65)$ obtidos assumiu o valor de 0,99. O item 6 (As informações no rótulo são suficientemente claras em relação à soja) foi aquele que apresentou diferença de 1,59 alocada acima do limite superior do diagrama $(1,17)$ (Figura 1). O procedimento

Tabela 3. Validação externa de um questionário de atitude com relação à soja e seus derivados dos clientes de unidades de alimentação e nutrição de uma empresa gráfica e de uma empresa de confecções, localizadas na cidade do Rio de Janeiro, (RJ), 2006.

\begin{tabular}{lc}
\hline $\begin{array}{l}\text { Questionário na UAN de EG versus } \\
\text { Questionário na UAN de EC }\end{array}$ & Escore \\
\hline Média das diferenças & $-0,09$ \\
Desvio-padrão das diferenças (d.p.d.) & $+0,64$ \\
Limites de concordância ( $p<0,05)$ & $-1,35$ a $+1,17$ \\
Desvio-padrão das médias (d.p.m.) & $+1,18$ \\
Razão dos desvios (d.p.d./d.p.m.) & 0,99 \\
\hline
\end{tabular}

Fonte: Altman \& Bland ${ }^{9}$.

UAN: unidades de alimentação e nutrição; EG: empresa gráfica; EC: empresa de confecções. recomenda a retirada do item. A Tabela 4 apresenta a tendência de concordância, discordância e estado de dúvida dos usuários em relação ao objeto da pesquisa.

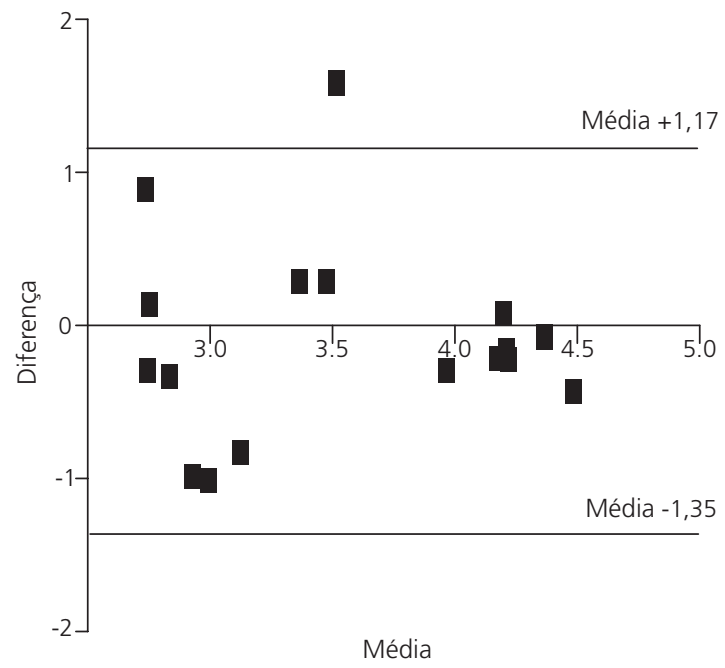

Figura 1. Escores obtidos na aplicação do questionário de atitude com relação à soja e seus derivados nos clientes de uma empresa gráfica e de uma empresa de confecções, localizadas na cidade do Rio de Janeiro (RJ), 2006.

Nota: UAN: unidade de alimentação e nutrição; EG: empresa gráfica: EC: empresa de confecções.

Tabela 4. Tendência de concordância, discordância e estado de dúvida dos clientes de unidades de alimentação e nutrição de uma empresa gráfica e de uma empresa de confecções localizadas na cidade do Rio de Janeiro (RJ), 2006.

\begin{tabular}{|c|c|c|c|c|c|c|}
\hline \multirow{2}{*}{ Categorias } & \multicolumn{2}{|c|}{ Concordância } & \multicolumn{2}{|c|}{ Discordância } & \multicolumn{2}{|c|}{ Estado de dúvida } \\
\hline & EG & EC & EG & EC & EG & EC \\
\hline \multirow{2}{*}{ Transgenia } & (18) 2,44 & - & (11) 3,82 & (11) 2,68 & (14) 2,71 & (14) 3,33 \\
\hline & & & & & & (18) 3,42 \\
\hline \multirow[t]{3}{*}{ Valor funcional } & (8) 3,18 & - & (6) 2,60 & (6) 2,89 & (10) 3,51 & (10) 3,22 \\
\hline & & & (19) 2,49 & & & (8) 2,29 \\
\hline & & & & & & (19) 3,50 \\
\hline \multirow[t]{2}{*}{ Comercialização } & (4) 3,82 & (4) 4,11 & - & - & - & - \\
\hline & (13) 4,27 & (13) 4,70 & & & & \\
\hline \multirow[t]{2}{*}{ Qualidade } & (3) 4,11 & (3) 4,33 & - & - & (17) 3,62 & (17) 3,54 \\
\hline & (20) 4,33 & (20) 4,40 & & & & \\
\hline \multirow[t]{3}{*}{ Rotulagem } & (1) 4,24 & (1) 4,16 & - & (7) 2,72 & - & - \\
\hline & (2) 4,13 & (2) 4,29 & & & & \\
\hline & (7) 4,31 & & & & & \\
\hline Valor nutricional & (9) 4,07 & (9) 4,28 & - & - & (15) 2,67 & (15) 3,00 \\
\hline
\end{tabular}

Número entre parênteses corresponde ao item do questionário; ausência de item com tendência de concordância, discordância ou estado de dúvida.

UAN: unidade de alimentação e nutrição; EG: empresa gráfica; EC: empresa de confecções. 


\section{I S C U S S Ã O}

Ragazzi ${ }^{11}$ destaca as vantagens atribuídas à escala do tipo Likert, tais como, maior facilidade quanto à sua elaboração e construção; o fato de ser um método que produz escalas mais homogêneas e aumenta a probabilidade de mensuração de atitudes unitárias; baseia-se em dados empíricos relacionados a respostas dos sujeitos, e permite maior amplitude de respostas a cada item. Estas foram as razões da escolha deste tipo de escala no presente estudo.

Baquero $^{12}$, Nunnally ${ }^{13}$ e Cronbach ${ }^{14}$ sugerem a construção de maior número possível de afirmações de início, para que a versão final da escala contenha número suficiente de itens. O questionário inicial, aplicado aos clientes da UAN de uma empresa de confecções, apesar de construído com 20 itens respondeu satisfatoriamente, uma vez que só foram retirados 3 itens. Por outro lado, Moriya et al. ${ }^{15}$ alertam que uma escala extensa pode ser cansativa, desmotivando os respondentes a exporem suas respostas reais.

Um instrumento precisa demonstrar confiabilidade e validade, antes de poder ser usado para medir uma variável de interesse específico. A validade e a confiabilidade são medidas que refletem a qualidade do instrumento; se o instrumento, então, não apresentar essas qualidades os resultados da pesquisa terão credibilidade limitada ou não terão utilidade prática. Ambas referem-se ao grau de consistência e precisão com que $\mathrm{o}$ instrumento mede a variável de interesse ${ }^{16}$ e até que ponto o faz ${ }^{17}$, ou seja, qual a coerência interna. Se um teste apresenta coerência interna considerável, ele é interpretável ${ }^{18}$. Estudos com o objetivo de validar instrumentos para medir aspectos comportamentais utilizam como coeficiente de confiabilidade o alfa de Cronbach.

No estudo de Behrens \& Da Silva', o questionário apresentou coeficiente de Cronbach igual a 0,71 , sendo um instrumento confiável para medir crenças, atitudes e hábitos de consumo com relação à soja e seus derivados.
Mendonça \& Tamayo ${ }^{19}$, com o objetivo de validar uma medida que aferisse a atitude de trabalhadores (313 sujeitos) em relação à retaliação organizacional, utilizaram uma escala de Likert contendo 12 proposições, das quais 6 relativas ao componente afetivo e 6 conativo. Os coeficientes de confiabilidade, avaliados pelo alfa de Cronbach, obtiveram 0,84 e 0,87 para fatores afetivo e conativo, respectivamente, demonstrando consistência interna da escala.

Formiga et al. ${ }^{20}$, com o objetivo de construir e validar um instrumento de medida direcionado à mensuração da intenção comportamental de praticar a caminhada, no mínimo, três vezes por semana, além de verificar o poder preditivo da Teoria de Ação Racional para tal comportamento, também utilizaram uma escala de Likert. O questionário contendo 21 proposições divididas em 3 seções: atitude, crenças comportamentais, crenças normativas, obtiveram alfa de Cronbach de 0,$80 ; 0,87 ; 0,76$, respectivamente, evidenciando a confiabilidade do instrumento.

Oliveira \& Neto ${ }^{21}$, com o objetivo de validar um instrumento útil para avaliar um quadro abrangente de atitudes ou reações frente à morte, utilizaram oito escalas breves sobre as diversas perspectivas sobre a morte, de autoria de Spilka et al. ${ }^{22}$, no formato Likert. A consistência interna das escalas, verificada pelo coeficiente alfa de Cronbach, deu os seguintes valores, respectivamente do $1^{\circ}$ ao $8^{\circ}$ factorial: $83,94,84,86,83$, $83,87,78$. Trata-se, assim, de uma escala octofactorial com boa fidelidade, possuindo boas propriedades psicométricas, podendo ser útil para avaliar as diversas perspectivas sobre a morte.

As escalas experimentadas com os clientes da UAN da empresa gráfica e os da empresa de confecções alcançaram alfa de Cronbach de 0,92 e 0,91 , respectivamente, valores superiores aos encontrados pelos diferentes autores. De acordo com Mueller?, uma escala que apresente coeficiente igual ou superior a 0,70 é um instrumento confiável para medir atitudes sobre o objeto relacionado. 
Vários estudos ${ }^{23-26}$ vêm utilizando o método de Altman \& Bland ${ }^{9}$ para verificar a concordância entre técnicas de mensuração. A justificativa é que o uso do coeficiente de correlação não mede a concordância, mede apenas a associação. Os autores sustentam que o uso da correlação está perdendo campo para uma abordagem alternativa, baseada em técnicas gráficas e cálculos simples. O diagrama de Altman \& Bland ${ }^{9}$ foi utilizado para verificar a concordância entre a matriz dos escores médios obtidos pela aplicação do questionário nas duas UAN. No procedimento sugerido pelos autores espera-se que $95 \%$ das diferenças estejam no intervalo de -2 a mais +2 desvios-padrão. Os resultados do presente estudo revelam 94,11\%. Este achado é devido à locação do item 6 fora dos limites de concordância. A EG obteve média de escores 4,31, enquanto a EC de 2,72. Este item abordava a clareza das informações contidas nos rótulos em relação à soja. As médias dispares mostram tendências opostas. Os demais itens da categoria rotulagem, que se referem à existência de desinteresse dos consumidores em verificar os ingredientes do rótulo, devido ao tamanho reduzido da letra e à não leitura habitual dos ingredientes da composição do produto no rótulo, receberam médias de escores 4,24 e 4,11 para a EG e 4,16 e 4,33 para a EC. Tais números refletem a concordância dos clientes para os itens em ambas as UAN.

Se o cliente não tem interesse e habitualidade em ler os ingredientes do rótulo, provavelmente não saberá se o produto contém soja. Por outro, as indústrias freqüentemente não utilizam a alegação funcional para soja permitida pela Resolução n 18 de 30 de abril de 1990 da ANVISA ${ }^{27}$, que poderia esclarecer o consumidor. Nesta hipótese, há razão suficiente para a discriminação do item 6 , porque esperava-se que a tendência fosse para o estado de dúvida e não para os extremos, como se aproximou a EC $(2,72)$.

Trabalho realizado por Moreira et al. ${ }^{24}$ encontrou razão dos desvios superiores a 0,60 para energia, colesterol e macronutrientes para ambos os sexos. O calculado na validação externa entre os clientes da UAN da empresa gráfica e os da empresa de confecções foi 0,99, resultado devido à discrepância assumida pelo item 6 , o que justifica a recomendação da retirada do item.

\section{ONCLUSÃO}

Os resultados permitem concluir que o questionário de atitude em relação à soja e seus derivados tem confiabilidade suficiente para avaliar a atitude de clientes da Alimentação Coletiva. A contribuição do estudo é colocar à disposição de pesquisadores um instrumento de medida de atitude do consumidor sobre um alimento, a soja, que vem se afirmando no hábito de consumo da população brasileira, seja de forma explícita ou implícita, como ingrediente de alimentos processados. O estudo requer atualidade, uma vez que pode aumentar a polêmica no âmbito da Segurança Alimentar e Nutricional.

\section{COLABORADORES}

S.M. COUTO participou da concepção do trabalho, do trabalho de campo, da pesquisa bibliográfica, da análise e da interpretação de dados. G.M.O. COELHO, A.S SOUZA e M.F. FERREIRA participaram do trabalho de campo e da análise de dados. V.A. MARIN participou da concepção do trabalho. H.S. LANZILLOTTI participou da concepção do trabalho, da análise e da interpretação de dados.

\section{REFER Ê N CIAS}

1. Behrens JH, Da Silva MAAP. Atitude do consumidor em relação à soja e produtos derivados. Ciênc Tecnol Aliment. 2004; 24 (3):431-9.

2. Costa NMB, Borëm A, Carvalho VF. Biotecnologia e nutrição. São Paulo: Nobel; 2003.

3. Giuliani GM. O dilema dos transgênicos. Estud Soc Agric. 2000; 15(1):13-38.

4. Araújo J. Produtos transgênicos na agricultura: questões técnicas, ideológicas e políticas. Cad Ciênc Tecnol. 2001; 8(1):1-18.

5. Brasil, Gabinete da Casa Civil. Decreto n. 4.680, de 24 de abril de 2003. Regulamenta o direito à 
informação, assegurado pela Lei $n^{\circ} 8.078$, de 11 de setembro de 1990, quanto aos alimentos e ingredientes alimentares destinados ao consumo humano ou animal que contenham ou sejam produzidos a partir de organismos geneticamente modificados, sem prejuízo do cumprimento das demais normas aplicáveis. D.O.U - Diário Oficial da União, Poder Executivo em 28 de abril de 2003, Seção 1, p.1. [acesso 2006 jun 20]. Disponível: $<$ www.ctnbio.gov.br>.

6. Freeman FS. Teorias práticas dos testes psicológicos. New York: Fundação Calousten Gulbenkian; 1962.

7. Müeller DJ. Measuring social attitudes: a handbook for researches and practioners. New York: Teachers College; 1986.

8. Siegel S. Estatística não paramétrica: para as ciências do comportamento. São Paulo: McGraw-Hill do Brasil; 1977.

9. Altman DG, Bland JM. Statistical methods for assessing agreement between two methods of clinical measurement. Lancet.1986; 1(8476): 307-10.

10. Delcourt C, Cubeau J, Balkau B, Papoz L. Codiab-Inserm-Zeneca Pharma Study Group. Limitions of the correlation coefficient in the validation of diet assessment methods. Epidemiol Res. 1994; 5(5): 518-24.

11. Ragazzi N. Uma escala de atitudes em relação à matemática [dissertação]. São Paulo: Universidade de São Paulo; 1976.

12. Baquero G. Testes psicométricos e projetivos: medidas psico-educacionais. São Paulo: Loyola; 1974.

13. Nunnaly JC. Introducción a la medición psicológica. México: McGraw-Hill; 1970.

14. Cronbach LJ. Essentials of psychological testing. New York: Harper and Row; 1970.

15. Moriya TM, Gir E, Hayashida M. Escala de atitude frente à AIDS: uma análise psicométrica. Rev Lat Am Enfermagem. 1994; 2(2):37-53.

16. Moran V, Wilk JR. Continuing education approval program: interrater reliability. J. Contin Educ Nurs. 1987; 18(2):44-6.

17. Anastasi A. Testes psicológicos. São Paulo: EPU; 1977.

18. Cronbach LJ. Coefficient alpha and the internal structure of the tests. New York: Psychometrika; 1951.
19. Mendonça H, Tamayo A. Construção e validação de um instrumento para a Medida de Atitude em Relação à Retaliação Organizacional (MARO). Aval Psicol. 2003; 2(2):147-53.

20. Formiga ASC, Dias MR, Saldanha AAW. Aspectos psicossociais da prevenção do infarto: construção e validação de um instrumento de medida. PsicoUSF. 2005; 10(1):31-40.

21. Oliveira JB, Neto F. Validação de um instrumento sobre diversas perspectivas da morte. J Anál Psicol. 2004; 22(2):355-67.

22. Spilka B, Stout L, Minton B, Sizemore D. Death and personal faith: a psychometric investigation. J Sci Study Relig. 1977; 16(2):169-78.

23. Guedes DP, Lopes CC, Guedes JERP. Reprodutibilidade e validade do questionário internacional de atividade física em adolescentes. Rev Bras Med Esporte. 2005; 11(2):151-8.

24. Moreira P, Sampaio D, Daniel M, De Almeida V. Validade relativa de um questionário de freqüência de consumo alimentar através da comparação com um registro alimentar de quatro dias. ACTA Médica Portuguesa. 2003; 16(1):412-20.

25. De Salvo VLMA, Gimeno SGA. Reprodutibilidade e validade do questionário de freqüência de consumo de alimentos. Rev Saúde Pública. 2002; 36(4):505-12.

26. Oliveira BG, Melendez JGV, Ciconelli RM, Rincón LG, Torres AAS, De Sousa LAP, et al. Versão em português, adaptação transcultural e validação de questionário para avaliação da qualidade de vida para pacientes portadores de marcapasso: AQUAREL. Arq Bras Cardiol. 2006; 87(2):75-83.

27. Brasil. Agência Nacional de Vigilância Sanitária. Resolução nº 18 de 30 de abril de 1999. Aprova o regulamento técnico que estabelece as diretrizes básicas para análise e comprovação de propriedades funcionais e ou de saúde alegadas em rotulagem de alimentos, constante do anexo desta portaria. D.O.U. - Diário Oficial da União; Poder Executivo, de 3 de maio de 1999 [acesso 2006 jun 20]. Disponível em: <http://www.anvisa.gov.br/ alimentos/legis/especifica/regutec.htm>.

Recebido em: 24/8/2007

Versão final reapresentada em: 2/4/2009 Aprovado em: 7/7/2009 


\section{ANEXO}

\section{QUESTIONÁRIO DE ATITUDE DOS CLIENTES DA ALIMENTAÇÃO COLETIVA EM RELAÇÃO À SOJA (ANTES DA VALIDAÇÃO)}

Instruções: as sentenças listadas a seguir expressam atitudes dos consumidores sobre a soja e produtos derivados. Leia cada sentença e, usando a escala abaixo, indique o seu grau de concordância ou discordância com cada item. Você deverá dar uma resposta para cada item do questionário.

POSITIVAS

5. Concordo muito

4. Concordo

3. Nem concordo/nem discordo

2. Discordo

1. Discordo muito

\section{NEGATIVAS}

5. Discordo muito

4. Discordo

3. Nem concordo/nem discordo

2. Concordo

1. Concordo muito

1. Há desinteresse dos consumidores em verificar os ingredientes nos rótulos devido ao tamanho reduzido da letra.

2. Habitualmente os consumidores não lêem os ingredientes da composição do produto no rótulo.

3. É preciso desenvolver produtos de soja com melhor sabor.

4. Existe muita diferença entre os sucos de frutas a base de soja e os tradicionais.

5. A difícil digestão da soja reduz seu consumo.

6. Produtos de soja podem não ser comprados por causarem flatulência.

7. As informações no rótulo são suficientemente claras em relação a soja.

8. Soja ajuda ao intestino funcionar bem.

9. Soja é uma boa fonte de proteínas.

10. O consumo de soja por mulheres ajuda a aliviar os sintomas da menopausa.

11. Deve-se evitar o consumo de produtos à base de soja transgênica.

12. Deveria ser obrigatório pela legislação brasileira, declarar no rótulo o uso de soja transgênica em produtos industrializados.

13. O consumo reduzido de soja e de produtos a base de soja deve-se a pouca divulgação de seus benefícios.

14. O óleo de soja é produzido a partir de soja transgênica.

15. Soja é melhor fonte de proteína que carne de animais (vaca, frango, peixe).

16. Os consumidores deveriam procurar as informações sobre os benefícios da soja na rotulagem.

17. O "leite" de soja é mais saudável que o leite de vaca por não conter hormônios.

18. A soja transgênica faz mal a saúde.

19. O consumo de soja ajuda a fortalecer os ossos.

20. Para os alérgicos a leite de vaca, o "leite" de soja é uma opção saudável. 
642 | S.M. couto et al.

Conclusão

\section{QUESTIONÁRIO DE ATITUDE DOS CLIENTES DA ALIMENTAÇÃO COLETIVA EM RELAÇÃO À SOJA (APÓS VALIDAÇÃO)}

Instruções: as sentenças listadas a seguir expressam atitudes dos consumidores sobre a soja e produtos derivados. Leia cada sentença e, usando a escala abaixo, indique o seu grau de concordância ou discordância com cada item. Você deverá dar uma resposta para cada item do questionário.

POSITIVAS

5. Concordo muito

4. Concordo

3. Nem concordo/nem discordo

2. Discordo

1. Discordo muito
NEGATIVAS

5. Discordo muito

4. Discordo

3. Nem concordo/nem discordo

2. Concordo

1. Concordo muito

1. Há desinteresse dos consumidores em verificar os ingredientes nos rótulos devido ao tamanho reduzido da letra.

2. Habitualmente os consumidores não lêem os ingredientes da composição do produto no rótulo.

3. É preciso desenvolver produtos de soja com melhor sabor

4. Existe muita diferença entre os sucos de frutas a base de soja e os tradicionais.

5. Produtos de soja podem não ser comprados por causarem flatulência

6. As informações no rótulo são suficientemente claras em relação a soja

7. Soja ajuda ao intestino funcionar bem.

8. Soja é uma boa fonte de proteínas.

9. O consumo de soja por mulheres ajuda a aliviar os sintomas da menopausa.

10. Deve-se evitar o consumo de produtos à base de soja transgênica.

11. O consumo reduzido de soja e derivados de soja deve-se a pouca divulgação de seus benefícios.

12. O óleo de soja é produzido a partir de soja transgênica.

13. Soja é melhor fonte de proteína que carne de animais (vaca, frango, peixe).

14. O "leite" de soja é mais saudável que o leite de vaca por não conter hormônios.

15. A soja transgênica faz mal a saúde.

16. O consumo de soja ajuda a fortalecer os ossos.

17. Para os alérgicos a leite de vaca, o "leite" de soja é uma opção saudável. 